Running Head: Aquatic exercise and multiple sclerosis

\title{
A randomized controlled trial to establish the impact of aquatic exercise training on functional capacity, balance, and perceptions of fatigue in female patients with multiple sclerosis
}

Mehdi Kargarfard $^{1}$, Ardalan Shariat ${ }^{2}$, Lee Ingle ${ }^{3}$, Leocani Letizia ${ }^{4}$, Mina Kargarfard ${ }^{5}$

${ }^{1}$ Department of Exercise Physiology, Faculty of Sport Sciences, University of Isfahan, Isfahan, Iran

${ }^{2}$ Department of Occupational Health, Faculty of Medicine and Health Sciences, University Putra Malaysia, Serdang, Malaysia

${ }^{3}$ Sport, Health \& Exercise Science, University of Hull, Kingston-upon-Hull, UK

${ }^{4}$ Neurological Department and INSPE-Institute of Experimental Neurology, Scientific Institute Hospital San Raffaele, Milan, Italy

${ }^{5}$ School of Dentistry, Isfahan University of Medical Sciences, Isfahan, Iran

ClinicalTrials.gov ID: NCT02882724

Running Head: Aquatic exercise and multiple sclerosis 


\section{ABSTRACT}

2 Objective: To assess the effects of 8-weeks aquatic exercise training on functional capacity, balance, 3 and perceptions of fatigue in women with multiple sclerosis (MS).

4 Design: A randomized controlled design.

5 Setting: Referral center of a multiple sclerosis society.

6 Participants: Women (age:36.4 \pm 8.2 ; BMI:24.5 \pm 1.9 ) diagnosed with RR-type (relapsing-remitting)

7 MS. After undergoing baseline testing, participants were allocated to either an intervention (aquatic 8 training programme) or a control group.

9 Interventions: The intervention consisted of an 8-week aquatic training programme (3 supervised 10 training sessions per week; session duration; 45-60 min; 50-75\% heart rate reserve).

11 Main measures: Six-minute walk test (6-MWT); balance (Berg Balance Scale; BBS), and 12 perceptions of fatigue (Modified Fatigue Impact Scale; MFIS), at baseline and after an 8 week 13 intervention. Differences over time between the experimental and control groups were assessed by a $142 \times 2$ (group by time) repeated measures analysis of variance (ANOVA).

15 Results: 32 women (age:36.4 \pm 8.2 ; BMI:24.5 \pm 1.9 ) completed the 8-week aquatic training 16 intervention (experimental group, $n=17$; controls, $n=15$ ). All outcome measures improved in the experimental group; 6-MWT performance ( $451 \pm 58 \mathrm{~m}$ to $503 \pm 57 \mathrm{~m} ; P<0.001$ ); BBS (pre-test mean, 53.59 \pm 1.70 ; post-test mean, 55.18 $\pm 1.18 ; P<0.001$ ), and in the MFIS (pre-test mean, $43.1 \pm 14.6$, posttest mean, $32.8 \pm 5.9 ; P<0.01)$. A significant group-by-time interaction was evident between the experimental and controls groups for 6-MWT:P<0.001, $\eta \mathrm{p}^{2}=0.551$; BBS: $P<0.001, \eta \mathrm{p}^{2}=0.423$; and MFIS: $P<0.001, \eta p^{2}=0.679$.

Conclusions: Aquatic exercise training improves functional capacity, balance, and perceptions of fatigue in women with MS.

Key Words: Aquatic; Exercise training; Functional capacity; Fatigue; Multiple sclerosis 
Multiple sclerosis (MS) is a neurodegenerative condition influencing the central nervous system, ${ }^{1}$ and is the third largest cause of adult neurological disabilities affecting an estimated 2.5 million adults worldwide. ${ }^{2}$ Approximately 400,000 young people in the $\mathrm{US}^{3}$ suffer from the condition, and prevalence rates increase by approximately 10,000 people every year. ${ }^{4}$ In recent years, studies in the Middle East and Iran indicate a relatively high prevalence of MS. In Iran, the average age of developing MS is 27 years, and $>40,000$ patients suffer from the condition. ${ }^{5}$

MS affects all aspects of a patient's life and causes a wide range of health, cognitive, and emotional problems. ${ }^{7}$ A number of associated symptoms including double vision, muscle weakness, fatigue and paralysis can all contribute to prolonged disability and a reduced quality of life. ${ }^{7,8}$ However, symptoms are highly variable; mobility problems, poor balance, and sensitivity to heat, are commonly experienced by patients. ${ }^{9}$ Mobility problems and poor balance are caused by a reduction in muscle strength, exercise tolerance, co-ordination, and reaction time which may increase the risk of falls and accidents. ${ }^{10}$ To curb the debilitating effects of MS, chronic exercise training is recommended to help control and improve symptoms. ${ }^{11}$ Patients with MS can improve their walking performance by improving their muscular strength and aerobic capacity. ${ }^{6,12,13}$ Indeed, exercise training benefits patients with MS in many ways, including improvements in cognitive skills and aerobic fitness, gradual improvement in depressive symptoms and physical exhaustion, and reduced risk of developing other significant ailments. ${ }^{14,15}$ Adaptations in muscular strength as a consequence of exercise training may lead to improved walking performance in people with MS. ${ }^{6}$ In a recent systematic review, aquatic exercise improved quality of life in affected patients with MS. The study showed as a consequence of aquatic training, $>60 \%$ of patients reported a very good or good quality of life, while in $<40 \%$ of patients, quality of life was reported to be fair/weak. However, there were no reports of any negative effects of water-based exercise. ${ }^{2}$

The role of aquatic exercise training has been championed by the American Physical Therapy Association (APTA) for a number of clinical conditions. ${ }^{16}$ Benefits include toning of muscles, improved aerobic capacity, improved flexibility, and improved anxiety levels. ${ }^{17}$ The natural buoyancy and viscosity of water can potentially provide a safe and protective environment for patients with MS to undertake exercise ${ }^{18}$ by making it feel easier to move their extremities without the fear of losing balance or falling over ${ }^{19}$ and often, these activities do not require a highly competent swimming technique to reap the benefits. ${ }^{20}$ Previous studies are yet to examine the impact

68 of chronic aquatic exercise training on both physical and psychological well-being including functional capacity, balance, and perceptions of fatigue in participants with MS. 
Females are an under-represented group in many exercise training studies, and little has been published regarding the impact of exercise training in females with MS. Likewise, MS data from previous studies has relied heavily on Caucasian populations. Hence the novelty of this project is in the population being investigated with mild MS (EDSS <3.5) focusing on outcome measures including functional capacity, balance, and perceptions of fatigue. The location of the study is also important; Isfahan, one of the major cities in Iran, has a soaring upward incidence and prevalence of MS which is the highest in Asia and Oceania ${ }^{5}$. Therefore, the aim of the study was to assess the effects of 8-weeks aquatic exercise training on functional capacity, balance, and perceptions of fatigue in Iranian women with MS. We hypothesized that 8-week of aquatic exercise training will significantly improve functional capacity, balance, and perceptions of fatigue in women with MS.

\section{METHODS}

The study was approved by the Ethics Committee of the University of Isfahan and the Isfahan University of Medical Sciences, Isfahan, Iran. All participants had been diagnosed with RR-type (relapsing-remitting) MS by Isfahan Multiple Sclerosis Society (IMSS) (a community-based group), and were screened from a rehabilitation program waiting list by a qualified neurologist. After screening, participants were randomly assigned based on age, distance walked during the 6-min walk test, and EDSS score to an intervention or control group. We used the Expanded Disability Status Scales (EDSS) questionnaire to measure the magnitude of neurological impairment and disability. The EDSS questionnaire ranges from a score of 0 (normal neuro status) to a score of 10 (death from MS). Participants with mild MS symptoms with a score $\leq 3.5$ were recruited to the study (Appendix 1). Participants were excluded if they had a relapse during the intervention and/or had developed any comorbidities during the intervention (Fig 1).

\section{Measurement of Outcomes}

Six-minute walk test (6-MWT); balance (Berg Balance Scale; BBS), and perceptions of fatigue

96 (Modified Fatigue Impact Scale; MFIS), were assessed at baseline and after 8 weeks in both groups

97 (Appendix 2). Testing was carried out by research assistants who were independent to the randomization process.

\section{Aquatic exercise training protocol}

100 The experimental group completed a program of physical training in water that included 8 101 consecutive weeks of 3 weekly sessions; each session consisted of 60 minutes of training between an 102 intensity of $50-75 \%$ of individual heart rate reserve. The session included a warm-up for 10 minutes, 
103 followed by 40 minutes of conditioning exercise, and the final 10 minutes acted as a cool-down

104 (Appendix 3).

105

106

107

\section{Statistical analysis}

108 Data normality was checked for all variables by the Kolmogorov-Smirnov test. On inspection, all variables were normally distributed. Baseline characteristics between groups were compared using an

110 independent t-test. The homogeneity of the variances were tested using the Levene's test (Appendix

111 4). Differences over time between the experimental and control groups were assessed by a 2x2 (group

112 by time) repeated measures analysis of variance (ANOVA). Bonferroni post hoc adjustments were 113 carried out where necessary, and partial eta ${ }^{2}\left(\eta_{\mathrm{p}}{ }^{2}\right)$ effect sizes were also calculated with 0.01, 0.06 and 1140.14 representing small, medium, and large effect sizes, respectively. ${ }^{22}$ We also performed an 115 intention to treat analysis (ITTA) using an imputation method, "last observation carried forward" 116 (LOCF) in order to deal with any missing data at follow up. ${ }^{23}$ Statistical analysis was performed using 117 SPSS v19 for Windows (IBM, New York, USA). An alpha level $<0.05$ was used as a threshold for 118 statistical significance. Values are presented as mean \pm standard deviation or $95 \%$ confidence 119 intervals (CI), unless otherwise specified.

\section{RESULTS}

123 Initially, 76 female participants with MS were eligible for the study, of which 40 agreed to participate following an explanation of the study details. In the study, 40 patients with MS were randomly assigned to either the experimental or control groups (20 participants per group).

126 However, 3 participants were excluded from the experimental group, and 5 participants were 127 excluded from the controls for non-medical reasons. Following exclusions, 32 female participants 128 (age: $36.4 \pm 8.2$; BMI: 24.5 \pm 1.9 ) completed the study (experimental group, $n=17$; controls, $n=15$ ). 129 All participants in the experimental group completed all of the training sessions. Physical 130 characteristics of both groups are reported in Table 1.

133 Participants in the intervention group (pre-test mean, $24.46 \pm 1.85 \mathrm{~kg} \cdot \mathrm{m}^{-2}$; post-test mean, $23.63 \pm 1.97$ $134 \mathrm{~kg} \cdot \mathrm{m}^{-2}$ ) decreased their BMI by $3.3 \%$, whilst controls (pre-test mean, $24.64 \pm 1.92 \mathrm{~kg} \cdot \mathrm{m}^{-2}$; post-test 135 mean, $26.07 \pm 2.42 \mathrm{~kg} \cdot \mathrm{m}^{-2}$ ) increased their BMI by 5.7\%. A significant group-by-time interaction $136 \quad\left(\mathrm{~F}_{1,30}=34.539, P<0.001, \eta \mathrm{p} 2=0.535\right)$ was evident between experimental and controls groups (Table 2$)$. 
141 The 6-MWT performance showed a significant increase from $451 \pm 58 \mathrm{~m}$ to $503 \pm 57 \mathrm{~m}(\mathrm{P}<0.001)$ in

142 the experimental group (12.2\% increase) after eight weeks of the aquatic exercise intervention.

143 Conversely, the mean distance for the 6-MWT decreased significantly from $447 \pm 30 \mathrm{~m}$ to $418 \pm 29 \mathrm{~m}$

$144(\mathrm{P}<0.01)$ in the controls $(6.3 \%$ decrease; Table 2$)$. A significant group-by-time interaction

$145\left(\mathrm{~F}_{1,30}=36.779, P=.001, \eta \mathrm{p} 2=0.551\right)$ was evident between experimental and controls groups.

\section{Sit to stand test}

148 A -19.2\% improvement in time to complete the sit-to-stand test was detected in the experimental 149 group (pre-test mean, 20.99 \pm 5.67 seconds; post-test mean, 16.82 \pm 5.10 seconds), whereas the control 150 group (pre-test mean, 21.35 \pm 4.70 ; post-test mean, $27.34 \pm 4.75$ ) worsened by $30.4 \%$. A significant 151 group-by-time interaction $\left(\mathrm{F}_{1,30}=80.094, P=.001, \eta \mathrm{p} 2=.728\right)$ was evident between experimental and 152 controls groups (Table 2).

\section{Push up test}

155 An improvement in performance in the push-up test was found in the experimental group (pre-test 156 mean, $16.94 \pm$ 9.13; post-test mean, $25.70 \pm 10.53$; $P<0.001$ ); whereas the control group (pre-test 157 mean, $18.07 \pm 7.13 n$; post-test mean, $10.20 \pm 5.03$; $P<0.001)$ worsened. A significant group-by-time 158 interaction $\left(F_{1,30}=39.816, P=0.001, \eta p 2=0.570\right)$ was evident between experimental and controls 159 groups (Table 2).

\section{Berg balance scale (BBS)}

162 In the experimental group, BBS score improved by 3.0\% (pre-test mean, $53.6 \pm 1.7$; post-test mean, $16355.2 \pm 1.2$ ), whereas performance in the controls deteriorated (pre-test mean, $52.3 \pm 3.3$; post-test mean, 50.2 \pm 4.6$)$. A significant group-by-time interaction $\left(\mathrm{F}_{1,30}=22.0, P=.001, \eta p 2=0.42\right)$ in $\mathrm{BBS}$ performance was evident between experimental and controls groups (Table 2).

\section{Total MFIS score}

168 In the experimental group, MFIS score improved by 20\% (pre-test mean, $43.1 \pm 14.6$, post-test mean, $16932.8 \pm 5.9$; $p<0.01$ ), whereas performance in the controls deteriorated (pre-test mean, 44.5 \pm 9.3 ; post170 test mean, $61.00 \pm 8.23 ; p<0.001)$. A significant group-by-time interaction $\left(F_{1,30}=63.461, p=0.001\right.$, $171 \eta \mathrm{p} 2=0.679$ ) in total MFIS score was evident between experimental and controls groups (Table 
172 2).Furthermore, similar trends were also noted for the sub-scales of the MFIS. A significant group-by173 time interaction $\left(F_{1,30}=62.090, p=0.001\right.$, $\left.\eta \mathrm{p} 2=0.674\right)$ was evident between experimental and 174 controls groups (Table 2) for the MFIS (physical) sub-scale, for the MFIS (cognitive) sub-scale ( $F_{1,30}$ $175=11.371, p=0.002, \eta p 2=0.274)$, and for the MFIS (psychosocial) sub-scale $\left(F_{1,30}=14.954, p=\right.$ $1760.002, \eta \mathrm{p} 2=0.333)$.

178 Differences over time between the experimental and control groups, at baseline and after 8 weeks intervention, are shown in fig 2.

\section{FIGURE 2 ABOUT HERE}

\section{DISCUSSION}

182 Our study examined the impact of an 8-week aquatic exercise training programme in 32 women with

183 MS in Isfahan, Iran. Lifestyle factors; diet; exposure to risk factors of cardiovascular disease; and 184 symptoms and signs of MS, including muscle weakness, fatigue, falls risk, cognitive dysfunction, and 185 paralysis can be different in developing nations. ${ }^{24}$ Impairment in neuromuscular function limits 186 functional and physiological ability, thereby leading to a progressive decrease in everyday activities 187 and a reduction in quality of life. ${ }^{25}$ The personal and economic costs of neurological disorders pose a significant burden on public health in these regions. ${ }^{26}$

189 In MS, aquatic exercise improves muscle strength. ${ }^{27}$ Musculoskeletal conditions including 190 osteoarthritis are frequently encountered problems in patients with MS. The effects of water 191 buoyancy reduce the loading on joints which can have a positive impact on symptoms. Swimming is 192 a non-weight-bearing activity associated with an increase in lean body mass but does not increase 193 bone mineral density (BMD).. ${ }^{28}$ This is unsurprising given that BMD responds over time to the 194 stress that it is placed under. In addition to the therapeutic effects of water buoyancy, the thermal 195 properties of water may also be key for improving symptoms in patients with MS. ${ }^{18}$

196 We found that there were significant improvements in functional capacity, balance, and perceptions 197 of fatigue compared to participants randomized to the control group. We believe that, this study has 198 novelty because it focuses specifically on an under-represented sub-group of clinical research, 199 women. In participants with MS, loss of balance is a significant issue due to poor judgment as well 200 as reduced power and motion control. ${ }^{12-13}$ Thus, risk of fractures from falls in participants with MS 201 is 2 to 3.5 times higher than in age-matched healthy controls. ${ }^{29}$ Therefore, interventions which can 202 help improve balance are fundamental in participants with MS. In our study, a significant 203 improvement in BBS score (balance) was found in the experimental group. These results are 204 supported by Salem et al. (2011) who showed an improvement in BBS score following aquatic 205 exercise training. ${ }^{30}$ No studies beyond 5 weeks are available, so our findings over a longer period (8 
weeks) are an original contribution to the knowledge base. A limitation of the BBS is that ceiling effects have been identified in higher performing individuals, ${ }^{31,32}$ limiting its applicability for all groups. Further, it does not evaluate verticality or cognitive factors affecting balance, ${ }^{33}$ which both influence risk of falling.

Smedal and colleagues (2006) conducted a study based on the Bobath concept which led to a significant improved in balance in participants with MS. ${ }^{34}$ Unfortunately, the study did not include a control group and included different outcome measures making study comparisons difficult with our own. Other studies have focused on participants with a wide range of symptoms (EDSS 1-6.6) but have not necessarily focused on the changes to balance in response to aquatic training. ${ }^{35}$ However, not all studies investigating improvements in balance in participants with MS have been positive. Debolt and co-workers (2004) assessed the effects of 8 weeks (24 sessions) of strength training on balance measures. ${ }^{36}$ They reported no change in balance over the intervention period. ${ }^{36}$ However, some participants demonstrated normal levels of balance at the start of the intervention period, therefore, it may be unsurprising that further improvements were not elicited. Clearly, though the type and mode of training stimulus will also be important for invoking positive physical adaptations.

We found that functional capacity measured by the 6-MWT significantly improved in participants with MS following the 8-week aquatic training intervention compared to controls. Previous studies ${ }^{6,37,38}$ have evaluated the effects of aerobic exercise training in participants with MS; and have shown significant improvements in functional capacity. Rampello and colleagues (2007) measured the impact of 8 weeks of aerobic exercise training on walking performance and maximal exercise capacity in 19 patients (14 female, 5 male) with mild to moderate MS. ${ }^{38}$ The aerobic training program consisted of 3 training sessions per week on a cycle ergometer. Each training session consisted of 30 minutes at $60 \%$ of maximum work rate. They found that aerobic exercise training was more effective than standardized neuro-rehabilitation (NR) therapy for improving walking performance and maximal exercise capacity. The favorable effects of exercise training in aquatic conditions for improving aerobic capacity has been established previously in apparently healthy older women ${ }^{39,40}$ but not women with MS and EDSS less than 3.5. Takeshima et al (2002) measured aerobic fitness in women aged 60-75 years pre- and following 12 weeks of water based exercise training. ${ }^{39}$ Women who completed $3 \mathrm{~d} \cdot \mathrm{wk}^{1}$ of a combination of resistance and endurance-type exercise (walking and dancing) achieved significant improvements in total cholesterol, exercise capacity, muscular strength, and percentage body fat.

Our findings add new insights. We have demonstrated that individualized aquatic exercise training can improve functional capacity, balance, and perceptions of fatigue in female participants with mild symptoms of MS (EDSS < 3.5). It should be noted that a previous study in 2015 in Iran showed a 
decrease in fatigue among women with MS is response to 8-week aquatic training, but used an EDSS score of less than 6 was as their inclusion criteria. ${ }^{41}$ Bayraktar et al. (2013) showed that comparing aquatic Tai-Chi exercises with home-based exercise showed a significant improvement in functional mobility during the 6-minute walk test and the Timed Up and Go Test $(P<0.05)$; no significant differences were observed in the home-based exercise group $(P>0.05)$. Aquatic exercises also provided statistically significant increases in gait speed. ${ }^{42}$ For aquatic-based programmes, water creates a buoyant, low-impact environment allowing participants to perform therapeutic exercise with less fear of falling. ${ }^{43}$ In addition, hydrostatic tension provides different proprioceptive and sensory feedback from that experienced on land. ${ }^{44}$

There are several limitations to our study. Firstly, the study was based on a relatively small number of participants who completed the aquatic exercise intervention. However, future studies with larger sample sizes, over a longer period of time, are needed to support or refute our findings. Secondly, the participants were limited to women, and only patients with EDSS less than or equal to 3.5. The clinicians' preference was not to include participants with more overt symptoms. Given the premise that heat sensitivity may limit exercise in patients with MS, it seems plausible that aquatic exercise training may be appropriate for patients with more pronounced progression of symptoms. It is unclear whether male participants would receive similar benefits to aquatic training, and further research needs to be conducted in this participant cohort. Further, randomized controlled trials comparing aquatic exercise training versus land-based aerobic training interventions versus mixed training regimes should be encouraged to determine the cumulative or additive benefits of these different environments on the physical and psychological well-being of participants with MS. We noted that the performance of our control group deteriorated significantly over the 8-week period. The control group did not perform any structured physical activity during the 8-week intervention which may have contributed to their deterioration. Furthermore, it is possible that the control group were less motivated during the post testing phase, hence contributing to their worsening performance at this time-point. However, we did try to somewhat mitigate for this eventuality by offering them potential involvement in future training studies. of fatigue in women with mild MS. In supervised conditions, the intervention is safe, and, based on our findings, should be investigated further in a larger cohort of patients, and in a wider spectrum of disease progression.

275

276 
280 1. Beck CA, Metz LM, Svenson LW, Patten SB. Regional variation of multiple sclerosis

2. Corvillo I, Varela E, Armijo F, Alvarez-Badillo A, Armijo O, Maraver F. Efficacy of aquatic therapy for multiple sclerosis: a systematic review. European journal of physical and rehabilitation medicine. 2017.

3. Marziniak M, Meuth SG, Gold R, Wiendl H. Multiple Sclerosis Update. Aktuelle Neurologie. 2011;38(04):190-202.

4. Motl RW, Snook EM. Physical activity, self-efficacy, and quality of life in multiple sclerosis. Annals of behavioral medicine. 2008;35(1):111-115.

5. Etemadifar M, Maghzi A-H. Sharp increase in the incidence and prevalence of multiple sclerosis in Isfahan, Iran. Multiple Sclerosis Journal. 2011;17(8):1022-1027.

6. Motl RW, Goldman MD, Benedict RH. Walking impairment in patients with multiple sclerosis: exercise training as a treatment option. Neuropsychiatr Dis Treat. 2010;6:767-774.

7. Auty A, Belanger C, Bouchard JP, et al. Burden of illness of multiple sclerosis: Part II: Quality of life. Canadian Journal of Neurological Sciences. 1998;25(1):31-38.

8. Motl RW, McAuley E. Symptom cluster and quality of life: preliminary evidence in multiple sclerosis. The Journal of neuroscience nursing: journal of the American Association of Neuroscience Nurses. 2010;42(4):212.

9. Comi G, Leocani L, Rossi P, Colombo B. Physiopathology and treatment of fatigue in multiple sclerosis. Journal of neurology. 2001;248(3):174-179.

10. Daubney ME, Culham EG. Lower-extremity muscle force and balance performance in adults aged 65 years and older. Physical therapy. 1999;79(12):1177.

11. Mostert S, Kesselring J. Effects of a short-term exercise training program on aerobic fitness, fatigue, health perception and activity level of subjects with multiple sclerosis. Multiple sclerosis. 2002;8(2):161-168.

12. Leroux A, Pinet H, Nadeau S. Task-oriented intervention in chronic stroke: changes in clinical and laboratory measures of balance and mobility. American journal of physical medicine \& rehabilitation. 2006;85(10):820-830.

13. Kaur J, Kumar A. Effect of task-specific training on gait parameters in hemiparetic stroke patients. IJPMR. 2009;20(1):23-26.

14. Marrie RA, Horwitz R, Cutter G, Tyry T, Campagnolo D, Vollmer T. High frequency of adverse health behaviors in multiple sclerosis. Multiple Sclerosis. 2009;15(1):105-113.

15. Beckerman H, de Groot V, Scholten MA, Kempen JCE, Lankhorst GJ. Physical activity behavior of people with multiple sclerosis: understanding how they can become more physically active. Physical therapy. 2010;90(7):1001-1013.

16. $\quad$ O’Sullivan SB, Schmitz TJ, Fulk G. Physical Rehabilitation. FA Davis; 2013.

17. Houdayer E, Comi G, Leocani L. The neurophysiologist perspective into MS plasticity. Frontiers in neurology. 2015;6.

18. Frohman AN, Okuda DT, Beh S, et al. Aquatic training in MS: neurotherapeutic impact upon quality of life. Annals of clinical and translational neurology. 2015;2(8):864-872.

19. Iucksch DD, Israel VL, Ribas DIR, Manffra EF. Gait characteristics of persons with incomplete spinal cord injury in shallow water. Journal of rehabilitation medicine. 2013;45(9):860-865.

20. Pearson M, Dieberg G, Smart N. Exercise as a therapy for improvement of walking ability in adults with multiple sclerosis: a meta-analysis. Archives of physical medicine and rehabilitation. 2015;96(7):1339-1348.

21. Chard S. Qualitative perspectives on aquatic exercise initiation and satisfaction among persons with multiple sclerosis. Disability and Rehabilitation. 2016:1-6.

22. Richardson JTE. Eta squared and partial eta squared as measures of effect size in educational research. Educational Research Review. 2011;6(2):135-147.

23. Heyting A, Tolboom J, Essers JGA. Statistical handling of drop-outs in longitudinal clinical trials. Statistics in medicine. 1992;11(16):2043-2061.

24. Vanhees L, McGee HM, Dugmore LD, Schepers D, van Daele P. A representative study of 
cardiac rehabilitation activities in European Union Member States: the Carinex survey. Journal of Cardiopulmonary Rehabilitation and Prevention. 2002;22(4):264-272.

25. Organization WH. Neurological Disorders: Public Health Challenges. World Health Organization; 2006.

26. Andlin-Sobocki P, Jönsson B, Wittchen H, Olesen J. Cost of disorders of the brain in Europe. European Journal of neurology. 2005;12(s1):1-27.

27. Gehlsen GM, Grigsby SA, Winant DM. Effects of an aquatic fitness program on the muscular strength and endurance of patients with multiple sclerosis. Physical therapy. 1984;64(5):653657.

28. Quiterio ALD, Carnero EA, Baptista FM, Sardinha LB. Skeletal mass in adolescent male athletes and nonathletes: relationships with high-impact sports. The Journal of Strength \& Conditioning Research. 2011;25(12):3439-3447.

29. Heesen C, Romberg A, Gold S, Schulz K-H. Physical exercise in multiple sclerosis: supportive care or a putative disease-modifying treatment. Expert review of neurotherapeutics. 2006;6(3):347-355.

30. Salem Y, Scott AH, Karpatkin H, et al. Community-based group aquatic programme for individuals with multiple sclerosis: a pilot study. Disability and rehabilitation. 2011;33(9):720-728.

31. Steffen T, Seney M. Test-retest reliability and minimal detectable change on balance and ambulation tests, the 36-item short-form health survey, and the unified Parkinson disease rating scale in people with parkinsonism. Physical therapy. 2008;88(6):733.

32. Lemay JF, Nadeau S. Standing balance assessment in ASIA D paraplegic and tetraplegic participants: concurrent validity of the Berg Balance Scale. Spinal cord. 2010;48(3):245-250.

33. Sibley KM, Beauchamp MK, Van Ooteghem K, Straus SE, Jaglal SB. Using the systems framework for postural control to analyze the components of balance evaluated in standardized balance measures: a scoping review. Archives of physical medicine and rehabilitation. 2015;96(1):122-132.

34. Smedal T, Lygren H, Myhr K, et al. Balance and gait improved in patients with MS after physiotherapy based on the Bobath concept. Physiotherapy Research International. 2006;11(2):104-116.

35. Bansi J, Bloch W, Gamper U, Kesselring J. Training in MS: influence of two different endurance training protocols (aquatic versus overland) on cytokine and neurotrophin concentrations during three week randomized controlled trial. Multiple Sclerosis Journal. 2013;19(5):613-621.

36. DeBolt LS, McCubbin JA. The effects of home-based resistance exercise on balance, power, and mobility in adults with multiple sclerosis. Archives of physical medicine and rehabilitation. 2004;85(2):290-297.

37. Kargarfard M, Eetemadifar M, Mehrabi M, Maghzi AH, Hayatbakhsh MR. Fatigue, depression, and health-related quality of life in patients with multiple sclerosis in Isfahan, Iran. European Journal of Neurology. 2012;19(3):431-437.

38. Rampello A, Franceschini M, Piepoli M, et al. Effect of aerobic training on walking capacity and maximal exercise tolerance in patients with multiple sclerosis: a randomized crossover controlled study. Physical therapy. 2007;87(5):545-555.

39. Takeshima N, Rogers ME, Watanabe E, et al. Water-based exercise improves health-related aspects of fitness in older women. Medicine and Science in Sports and Exercise. 2002;34(3):544-551.

40. Kravitz L, Mayo JJ. The physiological effects of aquatic exercise. Int J Med. 2006;332:305-11.

41. Razazian N, Yavari Z, Farnia V, et al. Exercising Impacts on Fatigue, Depression, and Paresthesia in Female Patients with Multiple Sclerosis. Medicine and science in sports and exercise. 2016;48(5):796-803.

42. Bayraktar D, Guclu-Gunduz A, Yazici G, et al. Effects of Ai-Chi on balance, functional mobility, strength and fatigue in patients with multiple sclerosis: a pilot study. NeuroRehabilitation. 2013;33(3):431-437.

43. Roth AE, Miller MG, Ricard M, Ritenour D, Chapman BL. Comparisons of static and dynamic balance following training in aquatic and land environments. Journal of Sport 
Rehabilitation. 2006;15(4):299-311.

44. Geigle PR, Cheek WL, Gould ML, Hunt HC, Shafiq B. Aquatic physical therapy for balance: the interaction of somatosensory and hydrodynamic principles. The Journal of Aquatic Physical Therapy. 1997;5(1):4-10. 